\title{
Development and psychometric testing of the active aging scale for Thai adults
}

This article was published in the following Dove Press journal:

Clinical Interventions in Aging

24 July 2014

Number of times this article has been viewed

\author{
Kattika Thanakwang ${ }^{1,2}$ \\ Sang-arun Isaramalai ${ }^{2,3}$ \\ Urai Hatthakit ${ }^{3}$ \\ 'Institute of Nursing, Suranaree \\ University of Technology, Nakhon \\ Ratchasima, Thailand; ${ }^{2}$ Research \\ Center for Caring System of Thai \\ Elderly, ${ }^{3}$ Faculty of Nursing, Prince \\ of Songkla University, Songkla, \\ Thailand
}

Background: Active aging is central to enhancing the quality of life for older adults, but its conceptualization is not often made explicit for Asian elderly people. Little is known about active aging in older Thai adults, and there has been no development of scales to measure the expression of active aging attributes.

Purpose: The aim of this study was to develop a culturally relevant composite scale of active aging for Thai adults (AAS-Thai) and to evaluate its reliability and validity.

Methods: Eight steps of scale development were followed: 1) using focus groups and in-depth interviews, 2) gathering input from existing studies, 3) developing preliminary quantitative measures, 4) reviewing for content validity by an expert panel, 5) conducting cognitive interviews, 6) pilot testing, 7) performing a nationwide survey, and 8) testing psychometric properties. In a nationwide survey, 500 subjects were randomly recruited using a stratified sampling technique. Statistical analyses included exploratory factor analysis, item analysis, and measures of internal consistency, concurrent validity, and test-retest reliability.

Results: Principal component factor analysis with varimax rotation resulted in a final 36-item scale consisting of seven factors of active aging: 1) being self-reliant, 2) being actively engaged with society, 3) developing spiritual wisdom, 4) building up financial security, 5) maintaining a healthy lifestyle, 6) engaging in active learning, and 7) strengthening family ties to ensure care in later life. These factors explained $69 \%$ of the total variance. Cronbach's alpha coefficient for the overall AAS-Thai was 0.95 and varied between 0.81 and 0.91 for the seven subscales. Concurrent validity and test-retest reliability were confirmed.

Conclusion: The AAS-Thai demonstrated acceptable overall validity and reliability for measuring the multidimensional attributes of active aging in a Thai context. This newly developed instrument is ready for use as a screening tool to assess active aging levels among older Thai adults in both community and clinical practice settings.

Keywords: active aging, scale development, psychometric evaluation, culturally sensitive measure, Thai elderly

\section{Introduction}

With a growing aging population worldwide, the World Health Organization (WHO) has devoted considerable effort to encouraging all countries to promote quality of life among older adults. ${ }^{1,2}$ As part of these efforts, the WHO has recently initiated a policy framework of active aging, defined as "the process of optimizing opportunities for health, participation, and security in order to enhance quality of life as people age". ${ }^{2}$ The policy framework builds upon the premise that the vast majority of people of all ages, especially older people, want to be active participants and contributors to society. The WHO argues that countries can afford to achieve quality of life for the aging population if governments, international organizations, and civil society enact "active aging" policies and programs that enhance the health, participation,
Correspondence: Kattika Thanakwang Institute of Nursing, Suranaree University of Technology, I I I University Avenue, Tambon, Suranaree, Amphur Muang, Nakhon Ratchasima 30000, Thailand Tel +66 44223520

Fax +6644223506

Email kattika@sut.ac.th 
and security of older citizens. ${ }^{2}$ However, active aging is a broad and internally complex notion. Although the concept is central to a global strategy for the management of aging populations, ${ }^{3,4}$ active aging has been defined in various ways across different countries and organizations. ${ }^{5}$ Countries need to utilize the WHO's active aging framework to conceptualize active aging and its components within the context of their own unique cultures and values.

The extent to which older persons are active or productive is of central interest to societies with growing numbers of older people, and the need to understand how to age actively or productively is a challenge to all countries. ${ }^{6}$ Understanding the processes associated with active aging has become a key focus for gerontological researchers. ${ }^{5,78}$ However, research on active aging has been plagued by a lack of consistency in the definition and measurement of the concept. ${ }^{9}$ This may stem from the multidimensional attributes of active aging, which depend upon a variety of influences or determinants surrounding elderly individuals, families, and nations. The lack of a consistent definition is reflected in the wide range of models and indicators found in the literature covering different approaches to the study of active aging.

Research on active aging has grown over recent decades, but theories continue to be based overwhelmingly on Western studies $^{9}$ that may not be applicable to ethnoculturally diverse societies, such as those in Asian countries. This is regrettable given the substantial cultural differences between East and West; for example, the strong emphasis on independence in the West as contrasted with Thai persons' acceptance of interdependence. ${ }^{10}$ The measurement of culturally-specific variables using measures developed in other parts of the world can be problematic due to differences in cultural contexts. The development of culturally-sensitive measures for research on aging in a particular context has proved challenging. ${ }^{11}$

There have been only a few studies on active aging in Thailand. Kespichayawattana and Wiwatvanich ${ }^{12}$ explored active aging attributes in elite Thai elderly adults, and Nantsupawat et $\mathrm{al}^{13}$ focused on active aging in older Thai adults living in one rural village in the northeastern region of the country. To our knowledge, little is known about active aging in lay older Thai adults. Specifically, there have been no attempts to develop scales to measure the expression of active aging in large samples, perhaps because of a lack of expertise in developing this type of instrument. Thus, a standard and culturally sensitive instrument, which covers multiple dimensions of active aging in Thai adults, needs to be developed.
The $\mathrm{WHO}^{2}$ has also suggested that active aging is influenced by cultural factors in addition to physical, cognitive, psychological, social, and environmental factors and economic resources. There is a need to explore how active aging might be defined and perceived within the cultural context of Thailand to illuminate those aspects of active aging that are unique versus those that are common across cultures. We attempt to add to the knowledge of active aging in older adults by identifying the processes involved with being actively engaged in life, and we present efforts to understand those components of active aging that are indigenous to older Thai adults. Such knowledge is essential to anyone involved in government agencies or scholarship research on aging, and should be considered a prerequisite for policy-making to promote active aging in Thailand.

Establishing the meaning and definition of active aging for Thai adults is an essential preliminary step towards identifying attributes for use in scale development. A standard and culturally sensitive instrument, which covers diverse dimensions of active aging in Thailand, is essential to assess levels of active aging. This type of scale would form the scientific basis for systematic assessment and intervention designed to enhance the active aging and quality of life of Thai older people. Therefore, this study aimed to develop a culturally relevant composite scale of active aging for Thai adults (AAS-Thai) and to evaluate its reliability and validity. We surveyed a large sample of older Thai adults and analyzed their perceptions and understanding of active aging using this new tool.

\section{Materials and methods}

The procedure we used to develop a comprehensive, culturally sensitive measure for older Thai adults was based on the multistep strategy outlined by Ingersoll-Dayton. ${ }^{11}$ The first step involved conducting focus groups and in-depth interviews. To identify culturally meaningful domains of active aging for elderly Thai adults, we used a qualitative approach to conduct focus groups and in-depth interviews with 64 older adults. This initial step identified six domains of active aging experienced by the participants (for more details, see Thanakwang et al). ${ }^{14}$

In the second step, we reviewed existing studies of active and positive aging, focusing particularly on studies of elderly Thai adults. ${ }^{8,12,13,15-17}$ We examined the literature for reference to factors of active aging similar to the six dimensions of active aging that had emerged from the interviews and focus groups. Combining the input from other studies with findings from the present study provided a comprehensive insight 
into active aging domains and facilitated the development of some closed-ended items.

The third step was to develop a preliminary quantitative measure, generating an item pool from the specified domains of active aging obtained. According to DeVellis, ${ }^{18}$ several processes are necessary to generate an item pool; for example, developing conceptual definitions of each specified domain, formulating operational definitions of the domains, identifying observable indicators of each domain, and constructing a blueprint of the item matrix. We incorporated words and phrases from the study participants' statements. The initial item pool consisted of 81 items within six domains.

In the fourth step, the 81 items were reviewed by a panel of seven experts specializing in multidisciplinary areas relevant to the study (ie, two experts on gerontological nursing, one on geriatric medicine, one on social gerontology, one on population development, one on linguistic and cultures, and one on instrument development). The experts were instructed to rate each item on a 4-point scale based on relevance and appropriateness, ranging from 1 (not relevant), 2 (somewhat relevant), 3 (quite relevant), to 4 (highly relevant). In addition, the experts were asked to evaluate the clarity and conciseness of the closed-ended items of the AAS-Thai by using "yes" or "no" responses on each item. They were also invited to suggest revised wordings for any items that seemed ambiguous, unclear, or inappropriate. The content validity of the measure was based on the expert concurrence using the content validity index (CVI), calculated for category evaluation and item evaluation. ${ }^{19}$ Values on the CVI greater than or equal to 0.80 indicated an acceptable content validity of the instrument. ${ }^{19}$ For this study, the overall CVI was 0.91 . The items rated at levels 3 or 4 were retained, whereas those rated at levels 1 or 2 by three or more experts were deleted or modified according to the experts' suggestions. In total, 21 items were deleted; the final questionnaire contained 60 items.

The fifth methodological step was to conduct cognitive interviews with ten active elderly Thai adults, who were members of the Health Promotion and Rehabilitation Center for the Elderly, Faculty of Nursing, Prince of Songkla University, Thailand. In recruiting these individuals, efforts were made to ensure that they had the capacity to think about the clarity of the closed-ended items from their own perspective as well as from the perspective of older Thai people with less education. ${ }^{11} \mathrm{~A}$ think-aloud question and a probing question were used in conjunction to explore participants' general perceptions as well as their reactions to specific aspects of the questions. The participants' review indicated that most of the items of the AAS-Thai were easy to understand by all participants, and only minor rewording was suggested. It was also noted that some words were redundant, and some questions were considered to be too long. Using data obtained from the respondents' perceptions and interpretations, problematic items with the potential to elicit response error were revised, ensuring the comprehensibility and practicality of the scale for lay older adults in Thailand.

The participants in our sample of ten suggested that the 5-level scale format was too long and quite difficult for older adults to respond to. Attributes of active aging do not, of course, relate solely to perceptions, but constitute the reality of everyday life. Therefore, in order to improve the clarity and practicality of the scale, the number of response categories was reduced to a 4-level scale format. We revised the response set to incorporate different degrees of truth (ranging from "not at all true" to "very true"), which was more easily understood by the respondents. The response choices appeared as 1 (not at all true), 2 (slightly true), 3 (somewhat true), and 4 (very true). This format was deemed most appropriate to measure the process of active aging in elderly people; it had fewer choices and no middle choice, which prevents middle-point choosing, a typical habit of elderly Thai adults. ${ }^{11}$ Finally, the third draft, 60 -item scale using a 4-point Likert type scale was finalized.

In step 6, the 60-item AAS-Thai was pilot-tested in one community using a convenience sample of 30 older adults. Preliminary psychometric testing with the 60 -item scale was carried out using item analysis. Three criteria were used in the process of deciding which items to retain: 1) a minimum interitem correlation of 0.20 and a maximum of $0.70,2)$ a minimum corrected item-total correlation coefficient of 0.30 , and 3 ) a minimum Cronbach's reliability of $0.70 .^{20,21}$ Five items (items $4,12,14,17$, and 32) were deleted since they had corrected item-total correlation coefficients of less than 0.30 . Three items (items 11, 13, and 34) were deleted because they had interitem correlations of less than 0.20. Furthermore, five items (items 28, 29, 43, 44, and 46) were removed since they had interitem correlations higher than 0.8 suggesting redundancy. This process culminated in a 47-item scale. The alpha coefficient for the overall scale was 0.97 , and the six subscales ranged from 0.81 to 0.92 , indicating good internal consistency. The corrected item-total correlations among the remaining 47 items were between 0.44 and 0.79 .

In steps 7 and 8 , this version of the AAS-Thai was tested by means of a nationwide survey, and further psychometric testing was conducted to examine the validity and reliability 
of this newly developed measure of active aging for Thai adults. The sample and setting, instrument, data collection, and data analysis are detailed in the next section.

\section{Sample and setting}

The sample size was chosen according to MacCallum et al's method of determining sample size in factor analysis. ${ }^{22}$ A minimum of five to ten samples per item is recommended for the psychometric evaluation of a new measure. ${ }^{19,21}$ Specifically, MacCallum et $\mathrm{al}^{22}$ recommend that a sample size of 500 or more in factor analytic studies is excellent. We therefore obtained a sample of 500 participants to test the scale. As our aim was to conduct a national survey, the sample was randomly selected from four regions of the country (central, north, northeast, and south) using the stratified sampling technique to make it representative to Thai older adults.

A five-stage random sampling method was used to select the study subjects:

1. Selecting provinces: one province of each region of Thailand (north, northeast, central, and south) was randomly selected. They were Nan (north), Nakhon Ratchasima (northeast), Kanchanaburi (central), and Songkla (south).

2. Selecting districts: one district of each province selected in stage one was randomly selected using a ballot method without replacement, yielding four districts for the study.

3. Selecting subdistricts: two subdistricts (or "tambons") of each selected district, one in an urban area (municipal) and another in a rural area (nonmunicipal), were selected by simple random sampling, resulting in eight subdistricts.

4. Selecting villages/communities: two villages/communities of each selected subdistrict were recruited using simple random sampling. This yielded 16 villages/communities; eight located in urban areas and eight in rural areas.

5. Selecting household and respondents: from a total number of older adults $(2,778)$ in 16 selected villages/ communities, the number of possible subjects was calculated proportional to the size of each village or community. The proportion sampled for this study was 0.18 (equal to $500 / 2,778$ ).

To select households according to the calculated number of the study sample in each village, we conducted systematic random sampling using household lists. One elderly respondent per household, who met the inclusion criteria, was selected. The inclusion criteria were 1) being an older person who is dwelling in the community, 2) being aged 60 years or older, 3 ) not suffering severe disabilities, severe dementia, or psychiatric disorders, and 4) being able to understand and speak Thai. If any household had more than one older person who met the inclusion criteria, simple random sampling was conducted by putting all their names in the pool and selecting one.

\section{Instruments}

The fourth draft of the AAS-Thai, which was revised after the pretesting step and included a demographic data form, was used as an instrument. The Healthy Aging Instrument (HAI) was used to examine construct validity.

The HAI was developed by Thiamwong et $\mathrm{al}^{23}$ for use with elderly Thai participants. The HAI consists of 35 items and a 4-point rating scale (1-4). There are nine components: 1) being self-sufficient and living simply, 2) managing stress, 3) having social relationships and support, 4) making merit and good deeds, 5) practicing self-care and self-awareness, 6) staying physically active, 7) staying cognitively active, 8) having social participation, and 9) accepting aging. ${ }^{23}$ The HAI demonstrates good validity and reliability (Cronbach's alpha $=0.88$ ).

\section{Procedure}

Prior to data collection, the research protocol was approved by the Institutional Review Board of the Faculty of Nursing, Prince of Songkla University, Thailand according to the tenets of the Declaration of Helsinki. Anonymity, privacy, and the right to withdraw from the study without negative consequences were guaranteed. Confidentiality was maintained in all data collecting and analysis processes, reports, and subsequent publications.

Data were gathered from January to April 2013. Faceto-face interviews were conducted in each respondent's home by researchers and trained interviewers. Before informed consent was given, respondents were informed of the overall purposes and protocols of the study and of the time required to complete it. Those participants who met all eligibility criteria were then fully enrolled in the study. The participants who were educated or well-read were asked to fill out a demographic sheet and to complete the questionnaire themselves. Researchers assisted nonliterate participants or those with any limitations by slowly reading the questionnaire aloud and then asking them to rate each item. 


\section{Statistical analyses}

Data were analyzed using the SPSS 14.0 software package for Windows (SPSS Inc., Chicago, IL, USA). Sociodemographic characteristics of the respondents were first analyzed using descriptive statistics. We calculated internal consistency of the scale and carried out an item analysis and a factor analysis.

Internal consistency reliability was examined by analyzing across items within the scale using internal consistency and item analysis. We used Cronbach's alpha coefficient to measure reliability for the overall scale and its subscales. A Cronbach's alpha value of 0.70 and above indicates sufficient internal consistency for a new tool. ${ }^{21}$

Item analysis was further performed to check whether the scale had acceptable internal consistency. This is one of the statistical techniques used to investigate the pattern of responses for each item of the scale, and it provides a basis for revisions to improve the effectiveness of test items and the validity of test scores. Following recommendations of the criteria for item analysis proposed by Ferketich ${ }^{20}$ and Nunnally and Bernstein, ${ }^{21}$ items of the AAS-Thai were examined, and decisions were made as to which items were to be retained, revised, or deleted.

Exploratory factor analysis (EFA) was used to condense and group highly correlated items together to create a new composite factor that represented each group of items. Before conducting EFA, the Kaiser-Meyer-Olkin value was obtained to determine whether the sample was adequate; we also carried out Bartlett's test of sphericity, which tests whether there is sufficient correlation between the variables and hence justification for the factor analysis. ${ }^{24} \mathrm{We}$ then performed an EFA using 1) factor extraction using principle component analysis and 2) factor rotation using the varimax method. An eigenvalue equal to or greater than 1.0 is considered as a criterion to determine the number of components to retain. ${ }^{24}$ Following the process of factor interpretation outlined by Hair et $\mathrm{al}^{24}$ we conducted the analysis in five steps. We first examined the factor matrix of loadings, focusing on the factor pattern matrix. It is recommended that factor loadings of 0.40 and higher have practical significance. We then examined the magnitude and significance of the loadings on each variable, and we also examined any cross-loadings. Thirdly, we assessed communalities (the amount of variance accounted for by the factor solution). A communality score of less than 0.50 was considered as not explaining enough variance. The fourth step was to respecify the factor model if necessary in the event of finding any one of several problems, for example, insignificant loadings, too many low communalities, and cross-loadings. The fifth step was to label the extracted factors according to the meaning of the items loading on them.

Construct validity was examined through concurrent validity. Concurrent validity examines the extent to which a measurement scale under development correlates with other scales that are designed to assess closely related constructs. ${ }^{25}$ As the concepts of healthy aging and active aging may be interconnected, ${ }^{2}$ we hypothesized that active aging is positively associated with healthy aging. Concurrent validity between AAS-Thai and HAI was assessed by Pearson's correlation coefficient.

We used a test-retest technique to evaluate the stability of the AAS-Thai. Data obtained from two different points in time were analyzed using Pearson's product moment correlation coefficient to estimate scale stability. The closer the coefficient is to 1.00 , the more stable the measurement. ${ }^{19}$

\section{Results \\ Sample characteristics}

The 500 participants were older Thai adults living in communities across four regions of Thailand. About two-thirds of them $(64 \%)$ were female. Ages of the sample ranged from 60 to 96 years, with a mean of 71 years (standard deviation $=7.88)$, and half of them $(50 \%)$ were young elderly, aged 60 to 69 years. Most of them (88\%) were Buddhist. More than half (54\%) of the subjects were married and living with their spouses. Most of them (69\%) had completed primary school (grade 4 ), and about $76 \%$ were able to read and write. The majority of them (52.8\%) were not working. With regard to economic status, about one-fourth of them (26\%) had a monthly income of 500-1,000 Baht, and nearly one-third (31\%) had an annual income lower than the poverty line (12,000 Baht). About $28 \%$ of them suffered from income insufficiency. More than half (54\%) lived in urban areas. The majority of them $(82 \%)$ coresided with adult children, and about $12 \%$ coresided with only a spouse.

\section{Psychometric analyses} Item analysis

The 47-item AAS-Thai was analyzed to examine the interitem correlation, intersubscale correlation, and item-total correlation. The results showed that the interitem correlations ranged from 0.18 to 0.74 , the intersubscale correlations ranged from 0.34 to 0.80 , and the item-total correlation coefficients ranged from 0.16 to 0.75 . However, two items 
(item 22 and 23) had item-total correlations of less than 0.30 and were removed. ${ }^{20,21}$ The 45 -item AAS-Thai was retained and reanalyzed. The results showed that the interitem correlations ranged from 0.27 to 0.74 , the intersubscale correlations ranged from 0.44 to 0.80 , and the item-total correlation coefficients ranged from 0.36 to 0.76 . The alpha coefficients of the six subscales ranged from 0.82 to 0.91 , and the reliability for the total scale was 0.96 , which is considered excellent.

\section{Exploratory factor analysis}

EFA using principal component analysis with varimax rotation was performed. The criteria used to consider the number of factors were 1) an eigenvalue greater than 1;2) scree plot characteristics; and 3) interpretability. ${ }^{21,24}$ Specifically, items were removed when 1) the item-factor loading was below $0.40 ; 2$ ) the loading(s) on each variable was (were) insignificant; 3 ) the communality score was less than 0.50 ; 4 ) the cross-loadings indicated relatively high loadings on more than one factor; and 5) the item did not contribute to factor interpretability. ${ }^{18,21,24}$ The results indicated that seven factors had a cumulative percentage of variance of $67.51 \%$. All factor loadings were greater than 0.40 with statistical significance. However, nine items (items 7, 14, 21, 27, 28, $34,39,40$, and 41) were eliminated since they had relatively high loadings on more than one factor. The remaining 36-item AAS-Thai draft was then reanalyzed.

The results of this last factor analysis with varimax rotation showed a Kaiser-Meyer-Olkin value of 0.933 , indicating sample adequacy for factor analysis. Bartlett's test of sphericity was significant $\left(\chi^{2}=12595.21, P<0.001\right)$, indicating the appropriateness of the data for further factor analysis. In the end, seven factors with eigenvalues greater than 1 were generated and 36 items retained. The eigenvalues ranged from 5.77 to 1.65 , and all components accounted for $68.53 \%$ of the total variance. The seven factors, corresponding eigenvalues, percentage of variances accounted for by each factor, and cumulative percentages of variance are presented in Table 1 . All of the 36 retained items had loading values greater than 0.40 on only one of the seven factors and could be meaningfully explained in terms of their corresponding components. The factor loadings ranged from 0.45 to 0.89 , with statistical significance $(P<0.001)$. The communality values ranged from 0.54 to 0.87 , indicating that the extracted factors explained a high proportion of item variance (see Table 2). The new seven factors of active aging were optimal, concise, theoretically organized, and presented a simple structure. These factors were named with respect to the process of being actively engaged in life: 1) being self-reliant, 2) being actively engaged with society, 3) developing spiritual wisdom, 4) building up financial security, 5) maintaining a healthy lifestyle, 6) engaging in active learning, and 7) strengthening family ties with respect to being cared for in late life.

The overall internal consistency reliability of the scale was 0.95 , indicating that it is a reliable instrument for measuring the multidimensional attributes of active aging. The Cronbach's alpha coefficients for the seven subscales ranged from 0.81 to 0.91 . The correlations between subscales ranged from 0.30 to 0.65 , and the correlations between the seven subscales and the entire scale ranged from 0.56 to 0.87 with statistical significance $(P<0.001)$, as shown in Table 3 .

\section{Concurrent validity}

Concurrent validity of the AAS-Thai was tested by examining possible relationships with the Healthy Aging Scale, a theoretically relevant scale, to compare the scale with previously established, conceptually related variables. With regard to the hypothesis that active aging is positively related

Table I Total loading, percent of variance, and cumulative percentage of the final draft 36-item AAS-Thai classified by eigenvalues greater than I

\begin{tabular}{|c|c|c|c|c|c|c|}
\hline \multirow[t]{2}{*}{ Factor } & \multicolumn{3}{|c|}{ Extraction sums of square loading } & \multicolumn{3}{|c|}{ Rotation extraction sums of square loadings } \\
\hline & Total & $\%$ of variance & Cumulative \% & Total & $\%$ of variance & Cumulative \% \\
\hline I & 13.834 & 38.427 & 38.427 & 5.773 & 16.037 & 16.037 \\
\hline 2 & 2.635 & 7.320 & 45.747 & 4.970 & 13.805 & 29.842 \\
\hline 3 & 2.401 & 6.670 & 52.417 & 3.577 & 9.936 & 39.778 \\
\hline 4 & $\mathrm{I} .737$ & 4.824 & 57.241 & 3.131 & 8.697 & 48.474 \\
\hline 5 & 1.650 & 4.583 & 61.824 & 2.865 & 7.960 & 56.434 \\
\hline 6 & 1.279 & 3.553 & 65.377 & 2.709 & 7.525 & 63.959 \\
\hline 7 & 1.135 & 3.154 & 68.531 & 1.646 & 4.572 & 68.531 \\
\hline
\end{tabular}

Notes: The seven factors of active aging: I, being self-reliant; 2, being actively engaged with society; 3 , developing spiritual wisdom; 4, building up financial security; 5, maintaining a healthy lifestyle; 6, engaging in active learning; 7, strengthening family ties to ensure care in later life.

Abbreviation: AAS-Thai, scale of active aging for Thai adults. 
Table 2 Factor loading of each item and factor of the 36-item AAS-Thai

\begin{tabular}{|c|c|c|c|c|c|c|c|c|}
\hline \multirow[t]{2}{*}{ Item } & \multicolumn{7}{|c|}{ Factor } & \multirow[t]{2}{*}{ Communality } \\
\hline & $I$ & 2 & 3 & 4 & 5 & 6 & 7 & \\
\hline I & 0.80 & & & & & & & $0.7 \mathrm{I}$ \\
\hline 2 & 0.76 & & & & & & & 0.69 \\
\hline II & 0.72 & & & & & & & 0.69 \\
\hline 9 & 0.68 & & & & & & & 0.68 \\
\hline 3 & 0.67 & & & & & & & 0.59 \\
\hline 13 & 0.65 & & & & & & & 0.65 \\
\hline 42 & 0.63 & & & & & & & 0.61 \\
\hline 15 & 0.55 & & & & & & & 0.55 \\
\hline 24 & & 0.74 & & & & & & 0.79 \\
\hline 26 & & 0.73 & & & & & & 0.62 \\
\hline 32 & & 0.73 & & & & & & 0.68 \\
\hline 25 & & 0.69 & & & & & & 0.79 \\
\hline 31 & & 0.69 & & & & & & 0.64 \\
\hline 30 & & 0.67 & & & & & & 0.62 \\
\hline 12 & & 0.62 & & & & & & 0.70 \\
\hline 29 & & 0.61 & & & & & & 0.65 \\
\hline 37 & & & 0.89 & & & & & 0.87 \\
\hline 38 & & & 0.87 & & & & & 0.82 \\
\hline 36 & & & 0.70 & & & & & 0.62 \\
\hline 33 & & & 0.61 & & & & & 0.59 \\
\hline 35 & & & 0.60 & & & & & 0.54 \\
\hline 44 & & & & 0.87 & & & & 0.87 \\
\hline 43 & & & & 0.84 & & & & 0.85 \\
\hline 45 & & & & 0.71 & & & & 0.66 \\
\hline 8 & & & & 0.48 & & & & 0.56 \\
\hline 19 & & & & & 0.81 & & & 0.69 \\
\hline 18 & & & & & 0.72 & & & 0.74 \\
\hline 20 & & & & & 0.71 & & & $0.7 \mathrm{I}$ \\
\hline 17 & & & & & 0.54 & & & 0.69 \\
\hline 16 & & & & & 0.45 & & & 0.66 \\
\hline 5 & & & & & & 0.78 & & 0.69 \\
\hline 4 & & & & & & 0.68 & & $0.7 \mathrm{I}$ \\
\hline 6 & & & & & & 0.60 & & 0.62 \\
\hline 10 & & & & & & 0.57 & & 0.66 \\
\hline 46 & & & & & & & 0.68 & 0.73 \\
\hline 47 & & & & & & & 0.68 & 0.72 \\
\hline
\end{tabular}

Notes: The seven factors of active aging: I, being self-reliant; 2, being actively engaged with society; 3 , developing spiritual wisdom; 4, building up financial security; 5 , maintaining a healthy lifestyle; 6 , engaging in active learning; 7 , strengthening family ties to ensure care in later life.

Abbreviation: AAS-Thai, scale of active aging for Thai adults.

Table 3 Correlation coefficients of subscale to subscale and subscale to entire scale, and alpha coefficients of the final draft 36-item AAS-Thai $(\mathrm{N}=500)$

\begin{tabular}{|c|c|c|c|c|c|c|c|c|c|}
\hline Scale & $\mathbf{I}$ & 2 & 3 & 4 & 5 & 6 & 7 & & Cronbach's alpha \\
\hline I. Being self-reliant & 1.00 & & & & & & & & 0.91 \\
\hline 2. Being actively engaged with society & $0.65^{*}$ & 1.00 & & & & & & & 0.91 \\
\hline 3. Developing spiritual wisdom & $0.46 *$ & $0.5 I^{*}$ & 1.00 & & & & & & 0.86 \\
\hline 4. Building up financial security & $0.58^{*}$ & $0.5 I^{*}$ & $0.43^{*}$ & 1.00 & & & & & 0.85 \\
\hline 5. Maintaining healthy lifestyle & $0.60 *$ & $0.50^{*}$ & $0.45 *$ & $0.48^{*}$ & 1.00 & & & & 0.81 \\
\hline 6. Engaging in active learning & $0.62^{*}$ & $0.63^{*}$ & $0.40 *$ & $0.45^{*}$ & $0.38^{*}$ & 1.00 & & & 0.82 \\
\hline $\begin{array}{l}\text { 7. Strengthening family ties to ensure } \\
\text { care in later life }\end{array}$ & $0.46^{*}$ & $0.36 *$ & $0.40^{*}$ & $0.50 *$ & $0.48 *$ & $0.30 *$ & 1.00 & & 0.85 \\
\hline 8. Entire scale & $0.87^{*}$ & $0.86 *$ & $0.67 *$ & $0.73 *$ & $0.7 I^{*}$ & $0.74 *$ & $0.56 *$ & 1.00 & 0.95 \\
\hline
\end{tabular}

Note: $* p<0.001$

Abbreviation: AAS-Thai, scale of active aging for Thai adults. 
Table 4 Pearson's correlation coefficients between the AAS-Thai and $\mathrm{HAl}(\mathrm{N}=30)$

\begin{tabular}{ll}
\hline Scale & HAI \\
\hline I. Being self-reliant & $0.64^{*}$ \\
2. Being actively engaged with society & $0.65^{*}$ \\
3. Developing spiritual wisdom & $0.6 I^{*}$ \\
4. Building up financial security & $0.64^{*}$ \\
5. Maintaining healthy lifestyle & $0.63^{*}$ \\
6. Engaging in active learning & $0.65^{*}$ \\
7. Strengthening family ties to ensure care in later life & $0.55^{*}$ \\
8. Entire scale & $0.84^{*}$ \\
\hline
\end{tabular}

Note: $* P<0.001$.

Abbreviations: AAS-Thai, scale of active aging for Thai adults; HAl, Healthy Aging Instrument.

to healthy aging, the findings indicated that the AAS-Thai had a significant positive association with the HAI $(r=0.84$, $P<0.001)$, and that the subscales of the AAS-Thai had significantly strong associations with the HAI $(r=0.55-0.65$, $P<0.001$ ), indicating the satisfactory construct validity of the AAS-Thai (see Table 4).

\section{Test-retest reliability}

To evaluate the stability of the final draft 36-item AAS-Thai, test-retest reliability within a 2-week interval was examined using 30 community-dwelling elderly participants. The mean score of the AAS-Thai at time one was 105.03, and the mean score at time two was 106.80 . The Pearson correlation coefficient between the two sets of scores was 0.92 , indicating good stability. In addition, the correlations of the seven subscales between time one and time two ranged from 0.78 to 0.92 (see Table 5). The results indicate that the 36 -item AAS-Thai is stable in terms of its test-retest reliability.

\section{Discussion}

This study attempted to highlight the importance of developing a culturally relevant measure of active aging within a Thai context as an alternative to importing standardized Western instruments that may not be relevant to Eastern cultures. The active aging scale for Thai adults (AAS-Thai) can be assumed to be a culturally sensitive instrument because the items emerged from the indigenous perspectives of lay older adults in Thailand.

\section{Seven factors of the AAS-Thai}

The factor analysis supported the proposition that the AASThai assesses the multidimensional nature of active aging or the process of being actively engaged in life.

The first important factor of active aging is being selfreliant, which is the factor that explains the greatest variance on the AAS-Thai. This factor included eight items representing a person's ability to independently take care of himself/ herself and includes tasks related to family-care (performing various activities in the household). From the perspective of older adults, being able to do what they wish is meaningful for their autonomy and implies that they are able to manage their lives on their own. ${ }^{26-28}$ Having meaningful activities in daily life that keep elderly individuals busy makes them proud that they are independent and not a burden on others. This notion is consistent with the valued concept of individualism among elderly Western people. ${ }^{10}$ Many Western scholars point out that active aging is based on the concept of selfhood, which assumes that elderly individuals must have self-responsibility and self-care for their own life. ${ }^{27,29}$ However, the findings of the present study expand upon the Western concept of self-reliance; among elderly Thai adults, this capacity is not only focused on self-caring but also incorporates the notion of caring for family. This rationale may come from the collectivistic nature of the Thai culture, in which people are considered to be fundamentally interdependent; therefore, the self is viewed as interconnected, and people are mutually responsible for one another. ${ }^{15,30}$ This finding is congruent with

Table 5 Stability estimates of the total scores for AAS-Thai in time one and time two

\begin{tabular}{|c|c|c|c|c|c|}
\hline \multirow[t]{2}{*}{ Scale } & \multicolumn{2}{|c|}{ AAS-Thai time one } & \multicolumn{2}{|c|}{ AAS-Thai time two } & \multirow[t]{2}{*}{$r$} \\
\hline & Mean & SD & Mean & SD & \\
\hline I. Being self-reliant & 27.57 & 4.96 & 28.00 & 4.00 & $0.88^{*}$ \\
\hline 2. Being actively engaged with society & 19.13 & 6.82 & 20.20 & 5.60 & $0.92 *$ \\
\hline 3. Developing spiritual wisdom & 16.27 & 2.03 & 16.07 & 1.93 & $0.8 I^{*}$ \\
\hline 4. Building up financial security & 9.53 & 3.74 & 9.40 & 3.35 & $0.90 *$ \\
\hline 5. Maintaining healthy lifestyle & 15.50 & 3.08 & 15.73 & 2.33 & $0.85^{*}$ \\
\hline 6. Engaging in active learning & 10.20 & 3.12 & 10.57 & 2.43 & $0.78^{*}$ \\
\hline $\begin{array}{l}\text { 7. Strengthening family ties to ensure } \\
\text { care in later life }\end{array}$ & 6.83 & 0.95 & 6.83 & 0.83 & $0.89 *$ \\
\hline 8. Entire scale & 105.03 & |8.7| & 106.80 & 14.23 & $0.92^{*}$ \\
\hline
\end{tabular}

Note: $* P<0.001$.

Abbreviations: AAS-Thai, scale of active aging for Thai adults; SD, standard deviation. 
research by Nantsupawat et a ${ }^{13}$ suggesting that active aging incorporates activities that benefit both elderly individuals and their families.

Being actively engaged with society (factor 2) incorporated eight items, including engaging in social participation and social contribution. These findings reflect the fact that elderly Thai adults perceive active aging as not just about being self-reliant but also about participating and contributing in the community and society at large. All countries require the participation of active or productive elderly people; in Thailand, this requirement is included in the national plan for older people. ${ }^{31}$ Maintaining socially active engagement is evident in a myriad of meaningful activities through which the elderly connect with others and contribute to the whole society. Therefore, productive engagement has been identified as an important indicator of active aging. Elderly people can make many social and economic contributions that are of great value to older people themselves, to their families, as well as to society at large. ${ }^{9,13,32}$ In other words, it can be said that active aging implies the utilization of older people's life competences as part of the human capital within society. ${ }^{33}$

Developing spiritual wisdom (factor 3) was also identified as an important aspect of active aging. This component involves having inner strength and calmness, including trusting in religion and making merits. It is widely agreed that spiritual growth is an important aspect of living a humanistic and meaningful life, which involves "going beyond", enabling one to contribute to a meaningful relationship with others and relating to God, religion, or transcendence. ${ }^{34}$ Ardelt $^{35}$ defines wisdom as an integration of cognitive, reflective, and affective (compassionate) characteristics in relation to understanding the truth of life, engaging in selfexamination to develop self-awareness and self-insight, and decreasing self-centeredness; the embodiment of these characteristics tends to result in compassionate love and concern for the welfare of others. The majority of elderly Thai people are Buddhist. The Thai people rely heavily on their Buddhist doctrines of doing good deeds as a way of "making merit". ${ }^{15,17}$ As one gets older, one has more wisdom and free time to be involved with higher forms of religious activities, engaging in mental development (meditation) and teaching the doctrine, or showing truth to others, activities that are considered as rendering more merit. In Thailand, it is not surprising that positive spirituality has been identified as the key indicator of healthy aging ${ }^{15-17}$ and active aging. ${ }^{8}$ This concept is considered by older Thai people to be an important component of active or productive aging. Active aging as defined by the $\mathrm{WHO}^{8}$ does not incorporate an aspect related to spiritual wisdom; thus, this component of active aging may be unique to elderly Thai people.

Building up financial security (factor 4) was characterized as preparing financially for later life and for funerary activities as well as having enough money for daily expenses. This component was extracted from the initial conceptual framework by EFA. This finding is congruent with numerous previous findings, suggesting that financial security has been associated with active aging, ${ }^{7,12}$ including successful aging. ${ }^{36}$ Being financially secure maximizes one's sense of security and autonomy. ${ }^{37}$ Numerous studies have indicated that financial security is considered crucial by elderly Thai adults. ${ }^{8,12,13,38}$ However, a study by Bowling ${ }^{5}$ argued that some elderly British participants also mentioned finances as a constituent of active aging. Thus, financial security may be significant for elderly Thai since most of them have limited income stability and depend on financial support from their children. ${ }^{38}$ Moreover, the importance of predeath preparations for financial security is probably uniquely found in research on elderly Thai people. In Thailand, elderly people prepare for their funerals by becoming a member of a community funeral fund to ensure that their children will have enough money to arrange their funerals. ${ }^{8,13}$

The five items that loaded on factor 5 (maintaining a healthy lifestyle) identified key aspects of health-promoting behaviors, such as eating healthy food and practicing physical activity or exercise. This suggests that those elderly adults who practice healthy lifestyles experience more active aging. This finding is consistent with numerous studies suggesting that a health promoting lifestyle has a strong association with healthy aging ${ }^{15,16}$ and successful aging. ${ }^{39}$ Bowling ${ }^{40}$ indicated that elderly British people also define active aging in terms of good physical health and fitness, identifying exercise as a key part of this.

Engaging in active learning (factor 6) consisted of four items reflecting the capacity of an elderly individual to learn about new information technologies to make them cognitively active and healthy. This finding suggests that engaging in active lifelong learning is important for older adults in Thailand, consistent with the findings of many prior studies that have confirmed that continued learning is important for active aging. ${ }^{7,27,41}$ At present, lifelong learning is imperative for older adults in an era of modernization, in which technologies are rapidly changing. There is a variety of ways for elderly people to extend their formal and informal learning over the course of their lives. ${ }^{14}$ Thus, policies to promote lifelong learning for older people in relation to their interests and circumstances should be established. 
The final factor of active aging that we identified was termed "Strengthening family ties to ensure care in later life" (factor 7). This consisted of two items: strengthening family ties and teaching children about filial piety. This dimension concerns activities engaged in by parents to ensure that their children will care for them when they are old or frail. This finding is congruent with a few studies in the Western context, ${ }^{7}$ but is more frequently the subject of research on Eastern cultures, ${ }^{37}$ particularly that of Thailand. ${ }^{8,13}$ It is prominently within the Thai context that elderly support is expected by family members, based on social norms such as filial piety and reciprocal exchanges. If children respect and care for older parents, it helps older parents feel that they have succeeded in nurturing and teaching their offspring, ${ }^{10,14}$ ensuring that they will be secure in later life.

\section{Psychometric properties of AAS-Thai}

The psychometric properties of the AAS-Thai were tested for two key issues: validity and reliability. With regard to the construct validity, factor analysis and concurrent validity were examined. For the reliability, internal consistency and test-retest reliability were calculated.

The results of the EFA indicated that the 7-factor AASThai with the retained 36 items is a well-constructed instrument for measuring active aging in Thai people. All items had loading values greater than 0.40 and loaded on only one factor, suggesting that the underlying factors are meaningful. The communalities were greater than 0.50 , indicating that the extracted factors satisfactorily explained the item variance. ${ }^{24}$ The eigenvalues of the seven factors ranged from 5.77 to 1.65 , and all components accounted for $68.53 \%$ of the total variance, which is greater than $50 \%$ of explained variance for the factors, ${ }^{24}$ indicating that the AAS-Thai is adequate for capturing the many of the attributes of active aging for an elderly Thai sample.

Our results supported the hypothesis that the AAS-Thai demonstrated a statistically significant association with the closely related constructs measured by the HAI. Hair et al ${ }^{24}$ suggested that the concurrent validity of a new instrument is acceptable when it correlates well with a criterion measure that has previously been validated. The findings indicate that the AAS-Thai was associated positively and significantly with HAI, and all seven of the active aging subscales were associated positively and significantly with the HAI, signifying a high correlation with the concept of healthy aging. This may be because health has been considered as one of the most important aspects of active aging. ${ }^{2,40}$

\section{Study limitations}

There are some limitations in this study that should be noted. First, the study results may be biased by participants responding in a socially desirable manner in order to present themselves in a positive light, particularly if they were interviewed in the presence of family members or neighbors. Future use of the AAS-Thai should incorporate simultaneous social desirability testing to confirm that these findings are not subject to bias. Second, this study did not compare standardized active aging or healthy aging scales developed for use with Western samples. Thus, we were unable to examine which factors are culturally specific and which are universally applicable across different cultures. A future comparison with a Western active aging scale would be useful to explore which dimensions of the AAS-Thai are unique and which are universal.

\section{Conclusion}

The AAS-Thai could potentially be the first culturally contextualized, relevant, and valid multidimensional scale of active aging in Thailand. Our study demonstrated that the 36-item AAS-Thai has satisfactory validity and reliability for assessing active aging levels among older Thai adults, suggesting that it could be used in both community and clinical practice settings.

\section{Acknowledgments}

$\mathrm{KT}$, SI, and UH conceived and designed the research. KT was responsible for data collection. KT and SI were responsible for data analysis. All authors contributed toward drafting and revising the manuscript and have approved the final version. The authors wish to thank Professor Dr Berit Ingersoll-Dayton, Associate Professor Dr Aranya Chowalit, and Assistant Professor Dr Wipavee Kong-in for their advice and their helpful comments on earlier versions of this manuscript.

\section{Disclosure}

The authors report no conflicts of interest in this work.

\section{References}

1. Walker A. A strategy for active aging. Int Soc Secur Rev. 2002;55(1): 121-139.

2. World Health Organization. Active aging: a policy framework. Geneva: World Health Organization, Noncommunicable Diseases and Mental Health Cluster, Noncommunicable Disease Prevention and Health Promotion Department, and Aging and Life Course; 2002. Available from: http://whqlibdoc.who.int/hq/2002/who_nmh_nph_02.8.pdf. Accessed September 8, 2013.

3. Clarke A, Warren L. Hopes, fears and expectations about the future: what do older people's stories tell us about active aging? Aging Soc. 2007;27: $465-488$. 
4. Walker A. Commentary: the emergence and application of active aging in Europe. J Aging Soc Policy. 2009;21(1):75-93.

5. Bowling A. Enhancing later life: how older people perceive active aging? Aging Ment Health. 2008;12(3):293-301.

6. Caro FG, Caspi E, Burr JA, Mutchler JE. Global activity motivation and activities of older people. Act Adapt Aging. 2009;33(3):191-208.

7. Buys L, Boulton-Lewis G, Tedman-Jones J, Edwards H, Knox M, Bigby C. Issues of active ageing: perceptions of older people with lifelong intellectual disability. Australas J Ageing. 2008;27(2):67-71.

8. Rattanamongkolgul D, Sritanyarat W, Manderson L. Preparing for aging among older villagers in northeastern Thailand. Nurs Health Sci. 2012;14(4):446-451.

9. Ranzijn R. Active ageing - another way to oppress marginalized and disadvantaged elders? Aboriginal Elders as a case study. $J$ Health Psychol. 2010;15(5):716-723.

10. Ingersoll-Dayton B, Saengtienchai $C$, Kespichayawattana J, Aungsuroch $Y$. Psychological well-being Asian style: the perspective of Thai elders. J Cross Cult Gerontol. 2001;16(3):283-302.

11. Ingersoll-Dayton $B$. The development of culturally-sensitive measures for research on ageing. Ageing Soc. 2011;31(3):355-370.

12. Kespichayawattana J, Wiwatvanich S. Active aging: a case study of elite Thai elderly. Bangkok: Thai Health Promotion Foundation; 2006. [Thai].

13. Nantsupawat W, Kamnuansilapa P, Sritanyarat W, Wongthanawasu S Family relationships, role and the meaning of active aging among rural northeastern Thai elders. Pacific Rim Int J Nurs Res. 2010;14(2): 137-148.

14. Thanakwang $\mathrm{K}$, Isaramalai $\mathrm{S}$, Hatthakit U. Thai cultural understanding of active aging from the perspectives of older adults: a qualitative study. Pacific Rim Int J Nurs Res. 2014;18(2):152-165.

15. Danyuthasilpe C, Amnatsatsue K, Tanasugarn C, Kerdmongkol P, Steckler AB. Ways of healthy aging: a case study of elderly people in a Northern Thai village. Health Promot Int. 2009;24(4):394-403.

16. Thanakwang K, Soonthorndhada K, Mongkolprasoet J. Perspectives on healthy aging among Thai elderly: a qualitative study. Nurs Health Sci. 2012;14(4):472-479.

17. Thiamwong L, McManus MS, Suwanno J. Development of the Thai healthy aging model: a grounded theory study. Nurs Health Sci. 2013 15(2):256-261.

18. DeVellis RF. Scale Development: Theory and Applications. 2nd ed. Thousand Oaks, CA: SAGE; 2003.

19. Burns N, Grove SK. The Practice of Nursing Research: Appraisal, Synthesis, and Generation of Evidence. 6th ed. St Louis, MO: Saunders; 2009.

20. Ferketich S. Focus on psychometrics: aspects of item analysis. Res Nurs Health. 1991;14(2):165-168.

21. Nunnally JC, Bernstein IH. Psychometric Theory. 3rd ed. New York, NY: McGraw-Hill; 1994.

22. MacCallum RC, Widaman KF, Zhang S, Hong S. Sample size in factor analysis. Psychol Methods. 1999;4(1):84-99.
23. Thiamwong L, Maneesriwongkul W, Malathum P, Jitapunkul S, Vorapongsathorn T, Stewart AL. Development and psychometric testing of the healthy aging instrument. Thai J Nurs Res. 2008;12(4): 285-296.

24. Hair JF Jr, Black WC, Babib BJ, Anderson RE, Tatham RL. Multivariate Data Analysis. 6th ed. Upper Saddle River, NJ: Prentice Hall; 2006.

25. McDowell I, Newell C. Measuring Health: A Guide to Rating Scales and Questionnaires. 2nd ed. New York, NY: Oxford University Press; 1996.

26. Clarke LH, Liu-Ambrose T, Zyla JA, McKay H, Khan Karim. "Being able to do the things that I want to do": older women with osteoporosis define health, quality of life, and well-being. Act Adapt Aging. 2005;29(4): 41-59.

27. Kwok JYC, Tsang KKM. Getting old with a good life: research on the everyday life patterns of active older people. Aging Int. 2012;37(3): 300-317.

28. Stenner $\mathrm{P}, \mathrm{McF}$ arquhar T, Bowling A. Older people and 'active ageing': subjective aspects of ageing actively. $J$ Health Psychol. 2011;16(3): $467-477$.

29. Katz S. Busy bodies: activity, aging, and the management of everyday life. J Aging Stud. 2000;14(2):135-152.

30. Ingersoll-Dayton B, Saengtienchai C, Kespichayawattana J, Aungsuroch Y. Measuring psychological well-being: insights from Thai elders. Gerontologist. 2004;44(5):596-604.

31. Jitapunkul S, Wivatvanit $\mathrm{S}$. National policies and programs for the aging population in Thailand. Aging Int. 2009;33:62-74.

32. Thanakwang K, Isaramalai SA. Productive engagement in older adults: a concept analysis. Nurs Health Sci. 2013;15(1):124-130.

33. Kruse A, Schmitt E. Generativity as a route to active ageing. Curr Gerontol Geriatr Res. 2012;2012:647650.

34. Dalby P. Is there a process of spiritual change or development associated with aging? A critical review of research. Aging Ment Health. 2006; 10(1):4-12.

35. Ardelt M. Development and empirical assessment of a three-dimensional wisdom scale. Res Aging. 2003;25(3):275-324.

36. Troutman M, Nies MA, Bentley M. Measuring successful aging in southern Black older adults. Educ Gerontol. 2011;37:38-50.

37. Chong AML, Ng SH, Woo J, Kwan AYH. Positive aging: the view of middle-aged and older adults in Hong Kong. Aging Soc. 2006;26(2): 243265.

38. Knodel J, Chayovan N. Intergenerational relationships and family care and support for Thai elderly. Aging Int. 2009;33:15-27.

39. Strawbridge WJ, Cohen RD, Shema SJ, Kaplan GA. Successful aging: predictors and associated activities. Am J Epidemiol. 1996; 144(2):135-141.

40. Bowling A. Perceptions of active ageing in Britain: divergences between minority ethnic and whole population samples. Age Ageing. 2009;38(6):703-710.

41. Boulton-Lewis GM. Education and learning for the elderly: why, how, what. Educ Gerontol. 2010;36:213-228.
Clinical Interventions in Aging

\section{Publish your work in this journal}

Clinical Interventions in Aging is an international, peer-reviewed journal focusing on evidence-based reports on the value or lack thereof of treatments intended to prevent or delay the onset of maladaptive correlates of aging in human beings. This journal is indexed on PubMed Central, MedLine,

\section{Dovepress}

CAS, Scopus and the Elsevier Bibliographic databases. The manuscript management system is completely online and includes a very quick and fair peer-review system, which is all easy to use. Visit http://www.dovepress. com/testimonials.php to read real quotes from published authors. 